\title{
Existence of Nontrivial Solutions for Periodic Schrödinger Equations with New Nonlinearities
}

\author{
Shaowei Chen and Dawei Zhang \\ School of Mathematical Sciences, Huaqiao University, Quanzhou 362021, China \\ Correspondence should be addressed to Shaowei Chen; swchen6@163.com \\ Received 13 April 2014; Accepted 29 May 2014; Published 15 June 2014 \\ Academic Editor: Mihai Mihǎilescu
}

Copyright (c) 2014 S. Chen and D. Zhang. This is an open access article distributed under the Creative Commons Attribution License, which permits unrestricted use, distribution, and reproduction in any medium, provided the original work is properly cited.

We study the Schrödinger equation: $-\Delta u+V(x) u+f(x, u)=0, u \in H^{1}\left(\mathbb{R}^{N}\right)$, where $V$ is 1-periodic and $f$ is 1-periodic in the $x$-variables; 0 is in a gap of the spectrum of the operator $-\Delta+V$. We prove that, under some new assumptions for $f$, this equation has a nontrivial solution. Our assumptions for the nonlinearity $f$ are very weak and greatly different from the known assumptions in the literature.

\section{Introduction and Statement of Results}

In this paper, we consider the following Schrödinger equation:

$$
-\Delta u+V(x) u+f(x, u)=0, \quad u \in H^{1}\left(\mathbb{R}^{N}\right),
$$

where $N \geq 1$. For $V$ and $f$, we assume the following.

(v) $V \in C\left(\mathbb{R}^{N}\right)$ is 1-periodic in $x_{j}$ for $j=1, \ldots, N, 0$ is in a spectral gap $\left(-\mu_{-1}, \mu_{1}\right)$ of $-\Delta+V$, and $-\mu_{-1}$ and $\mu_{1}$ lie in the essential spectrum of $-\Delta+V$.

Denote

$$
\mu_{0}:=\min \left\{\mu_{-1}, \mu_{1}\right\} .
$$

$\left(\mathbf{f}_{\mathbf{1}}\right) f \in C\left(\mathbb{R}^{N} \times \mathbb{R}\right)$ is 1-periodic in $x_{j}$ for $j=1, \ldots, N$. And there exist constants $C>0$ and $2<p<2^{*}$ such that

$|f(x, t)| \leq C\left(1+|t|^{p-1}\right), \quad \forall(x, t) \in \mathbb{R}^{N} \times \mathbb{R}$,

where

$$
2^{*}:= \begin{cases}\frac{2 N}{(N-2)}, & N \geq 3 \\ \infty, & N=1,2 .\end{cases}
$$


for $x \in \mathbb{R}^{N}$. And there exists $D>0$ such that

$$
\inf _{x \in \mathbb{R}^{N},|t| \geq D} \frac{f(x, t)}{t}>\max _{\mathbb{R}^{N}} V_{-},
$$

where $V_{ \pm}(x)=\max \{ \pm V(x), 0\}, \forall x \in \mathbb{R}^{N}$.

$\left(\mathbf{f}_{3}\right)$ For any $(x, t) \in \mathbb{R}^{N} \times \mathbb{R}, \widetilde{F}(x, t) \geq 0$, where

$$
\widetilde{F}(x, t):=\frac{1}{2} t f(x, t)-F(x, t), \quad F(x, t)=\int_{0}^{t} f(x, s) d s .
$$

$\left(\mathbf{f}_{4}\right)$ There exist $0<\kappa<D$ and $v \in\left(0, \mu_{0}\right)$ such that, for every $(x, t) \in \mathbb{R}^{N} \times \mathbb{R}$ with $|t|<\kappa$,

$$
|f(x, t)| \leq v|t|
$$

and, for every $(x, t) \in \mathbb{R}^{N} \times \mathbb{R}$ with $\kappa \leq|t| \leq D$,

$$
\widetilde{F}(x, t)>0 .
$$

Remark 1 . By the definitions of $F$ and $\widetilde{F}$, it is easy to verify that, for all $(x, t) \in \mathbb{R}^{N} \times(\mathbb{R} \backslash\{0\})$,

$$
\frac{\partial}{\partial t}\left(\frac{F(x, t)}{t^{2}}\right)=\frac{2 \widetilde{F}(x, t)}{t^{3}} .
$$


Together with $f(x, t)=o(t)$ as $|t| \rightarrow 0$ and $\left(\mathbf{f}_{\mathbf{3}}\right)$, this implies that

$$
F(x, t) \geq 0 \quad \forall(x, t) \in \mathbb{R}^{N} \times \mathbb{R} .
$$

Remark 2. There are many functions satisfying $\left(\mathbf{f}_{\mathbf{1}}\right)-\left(\mathbf{f}_{\mathbf{4}}\right)$. We give several examples here.

Example 1. $D=1+\mu_{0} / 2+e^{1+\max _{\mathbb{R}^{N}} V_{-}}, \kappa=1+\mu_{0} / 2, \nu=\mu_{0} / 2$, and

$$
f(x, t)= \begin{cases}0, & |t| \leq 1 \\ t \ln |t|, & |t|>1\end{cases}
$$

Example 2. $D=3+\mu_{0} / 2+2 \max _{\mathbb{R}^{N}} V_{-}, \kappa=3 / 2, \nu=\mu_{0} / 2$, and

$$
f(x, t)= \begin{cases}0, & |t| \leq 1, \\ D(t-1), & t>1, \\ D(t+1), & t<-1 .\end{cases}
$$

Example 3. $D=\mu_{0} / 2+e^{1+\max _{\mathbb{R}^{N}} V_{-}}, \kappa=v=\mu_{0} / 2$, and $f(x, t)=t \ln (1+|t|)$.

A solution $u$ of (1) is called nontrivial if $u \neq \equiv 0$. Our main results are as follows.

Theorem 3. Suppose $(\mathbf{v})$ and $\left(\mathbf{f}_{\mathbf{1}}\right)-\left(\mathbf{f}_{\mathbf{4}}\right)$ are satisfied. Then (1) has a nontrivial solution.

Note that

$\left(\mathbf{f}_{2}^{\prime}\right)$ the $\operatorname{limits}_{\lim _{t \rightarrow 0}} f(x, t) / t=0$ and $\lim _{|t| \rightarrow \infty}(f(x$, $t) / t)=+\infty$ hold uniformly for $x \in \mathbb{R}^{N}$.

Implying $\left(\mathbf{f}_{\mathbf{2}}\right)$, we have the following corollary.

Corollary 4. Suppose $(\mathbf{v}),\left(\mathbf{f}_{1}\right),\left(\mathbf{f}_{2}^{\prime}\right),\left(\mathbf{f}_{3}\right)$, and $\left(\mathbf{f}_{4}\right)$ are satisfied. Then (1) has a nontrivial solution.

It is easy to verify that the condition

$$
\left(\mathbf{f}_{4}^{\prime}\right) \widetilde{F}(x, t)>0 \text {, for every }(x, t) \in \mathbb{R}^{N} \times \mathbb{R} \text {. }
$$

And the assumption that $f(x, t) / t \rightarrow 0$ as $t \rightarrow 0$ uniformly for $x \in \mathbb{R}^{N}$ imply $\left(\mathbf{f}_{\mathbf{3}}\right)$ and $\left(\mathbf{f}_{4}\right)$. Therefore, we have the following corollary.

Corollary 5. Suppose $(\mathbf{v}),\left(\mathbf{f}_{1}\right),\left(\mathbf{f}_{2}\right)$, and $\left(\mathbf{f}_{\mathbf{4}}^{\prime}\right)$ are satisfied. Then (1) has a nontrivial solution.

Semilinear Schrödinger equations with periodic coefficients have attracted much attention in recent years due to its numerous applications. One can see [1-24] and the references therein. In [2], the authors used the dual variational method to obtain a nontrivial solution of (1) with $f(x, t)=$ $\pm W(x)|t|^{p-2} t$, where $W$ is an asymptotically periodic function. In [20], Troestler and Willem firstly obtained nontrivial solutions for (1) with $f$ being a $C^{1}$ function satisfying the Ambrosetti-Rabinowitz condition:

(AR) there exists $\alpha>2$ such that, for every $u \neq 0,0<$ $\alpha G(x, u) \leq g(x, u) u$, where $g(x, u)=-f(x, u), G(x$, $u)=-F(x, u)$, and

$$
\left|\frac{\partial f(x, u)}{\partial u}\right| \leq C\left(|u|^{p-2}+|u|^{q-2}\right)
$$

with $2<p<q<2^{*}$. Then, in [9], Kryszewski and Szulkin developed some infinite-dimensional linking theorems. Using these theorems, they improved Troestler and Willem's results and obtained nontrivial solutions for (1) with $f$ only satisfying $\left(\mathbf{f}_{\mathbf{1}}\right)$ and the (AR) condition. These generalized linking theorems were also used by Li and Szulkin to obtain nontrivial solution for (1) under some asymptotically linear assumptions for $f$ (see [11]). In [13] (see also [14]), existence of nontrivial solutions for (1) under $\left(\mathbf{f}_{1}\right)$ and the (AR) condition was also obtained by Pankov and Pflüger through approximating (1) by a sequence of equations defined in bounded domains. In the celebrated paper [17], Schechter and Zou combined a generalized linking theorem with the monotonicity methods of Jeanjean (see [8]). They obtained a nontrivial solution of (1) when $f$ exhibits the critical growth. A similar approach was applied by Szulkin and Zou to obtain homoclinic orbits of asymptotically linear Hamiltonian systems (see [19]). Moreover, in [5] (see also [6]), Ding and Lee obtained nontrivial solutions for (1) under some new superlinear assumptions on $f$ different from the classical (AR) conditions.

Our assumptions on $f$ are very weak and greatly different from the assumptions mentioned above. In fact, our assumptions $\left(\mathbf{f}_{\mathbf{1}}\right)-\left(\mathbf{f}_{\mathbf{4}}\right)$ do not involve the properties of $f$ at infinity. It may be asymptotically linear growth at infinity,

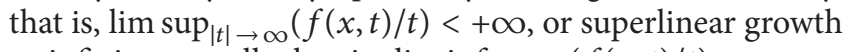
at infinity as well, that is, $\liminf _{|t| \rightarrow \infty}(f(x, t) / t)=+\infty$. Moreover, the assumptions $\left(\mathbf{f}_{\mathbf{1}}\right)-\left(\mathbf{f}_{4}\right)$ allow $f(x, t) \equiv 0$ in a neighborhood of $t=0$ (see Remark 2).

In this paper, we use the generalized linking theorem for a class of parameter-dependent functionals (see [17, Theorem 2.1] or Proposition 8 in the present paper) to obtain a sequence of approximate solutions for (1). Then, we prove that these approximate solutions are bounded in $L^{\infty}\left(\mathbb{R}^{N}\right)$ and $H^{1}\left(\mathbb{R}^{N}\right)$ (see Lemmas 13 and 14). Finally, using the concentration-compactness principle, we obtain a nontrivial solution of (1).

Notation. $B_{r}(a)$ denotes the open ball of radius $r$ and center $a$. For a Banach space $E$, we denote the dual space of $E$ by $E^{\prime}$ and denote strong and weak convergence in $E$ by $\rightarrow$ and $\rightarrow$, respectively. For $\varphi \in C^{1}(E ; \mathbb{R})$, we denote the Fréchet derivative of $\varphi$ at $u$ by $\varphi^{\prime}(u)$. The Gateaux derivative of $\varphi$ is denoted by $\left\langle\varphi^{\prime}(u), v\right\rangle, \forall u, v \in E . L^{p}\left(\mathbb{R}^{N}\right)$ denotes the standard $L^{p}$ space $(1 \leq p \leq \infty)$, and $H^{1}\left(\mathbb{R}^{N}\right)$ denotes 
the standard Sobolev space with norm $\|u\|_{H^{1}}=\left(\int_{\mathbb{R}^{N}}\left(|\nabla u|^{2}+\right.\right.$ $\left.\left.u^{2}\right) d x\right)^{1 / 2}$. We use $O(h), o(h)$ to mean $|O(h)| \leq C|h|$, $o(h) /|h| \rightarrow 0$.

\section{Existence of Approximate Solutions for (1)}

Under the assumptions $(\mathbf{v}),\left(\mathbf{f}_{1}\right)$, and $\left(\mathbf{f}_{2}\right)$, the functional

$$
\begin{aligned}
\Phi(u)= & \frac{1}{2} \int_{\mathbb{R}^{N}}|\nabla u|^{2} d x+\frac{1}{2} \int_{\mathbb{R}^{N}} V(x) u^{2} d x \\
& +\int_{\mathbb{R}^{N}} F(x, u) d x
\end{aligned}
$$

is of class $C^{1}$ on $X:=H^{1}\left(\mathbb{R}^{N}\right)$, and the critical points of $\Phi$ are weak solutions of (1).

There is a standard variational setting for the quadratic form $\int_{\mathbb{R}^{N}}\left(|\nabla u|^{2}+V(x) u^{2}\right) d x$. For the reader's convenience, we state it here. One can consult [5] or [6] for more details.

Assume that (v) holds, and let $S=-\Delta+V$ be the self-adjoint operator acting on $L^{2}\left(\mathbb{R}^{N}\right)$ with domain $D(S)=H^{2}\left(\mathbb{R}^{N}\right)$. By virtue of $(\mathbf{v})$, we have the orthogonal decomposition

$$
L^{2}=L^{2}\left(\mathbb{R}^{N}\right)=L^{+}+L^{-}
$$

such that $S$ is negative (resp., positive) in $L^{-}$(resp., in $L^{+}$). As in [5, Section 2] (see also [6, Chapter 6.2]), let $X=D\left(|S|^{1 / 2}\right.$ ) be equipped with the inner product

$$
(u, v)=\left(|S|^{1 / 2} u,|S|^{1 / 2} v\right)_{L^{2}}
$$

and norm $\|u\|=\left\||S|^{1 / 2} u\right\|_{L^{2}}$, where $(\cdot, \cdot)_{L^{2}}$ denotes the inner product of $L^{2}$. From (v),

$$
X=H^{1}\left(\mathbb{R}^{N}\right)
$$

with equivalent norms. Therefore, $X$ continuously embeds in $L^{q}\left(\mathbb{R}^{N}\right)$ for all $2 \leq q \leq 2 N /(N-2)$ if $N \geq 3$ and for all $q \geq 2$ if $N=1,2$. In addition, we have the decomposition

$$
X=X^{+}+X^{-} \text {, }
$$

where $X^{ \pm}=X \cap L^{ \pm}$is orthogonal with respect to both $(\cdot, \cdot)_{L^{2}}$ and $(\cdot, \cdot)$. Therefore, for every $u \in X$, there is a unique decomposition

$$
u=u^{+}+u^{-}, \quad u^{ \pm} \in X^{ \pm}
$$

with $\left(u^{+}, u^{-}\right)=0$ and

$$
\int_{\mathbb{R}^{N}}|\nabla u|^{2} d x+\int_{\mathbb{R}^{N}} V(x) u^{2} d x=\left\|u^{+}\right\|^{2}-\left\|u^{-}\right\|^{2}, \quad u \in X .
$$

Moreover,

$$
\begin{gathered}
\mu_{-1}\left\|u^{-}\right\|_{L^{2}}^{2} \leq\left\|u^{-}\right\|^{2}, \quad \forall u \in X, \\
\mu_{1}\left\|u^{+}\right\|_{L^{2}}^{2} \leq\left\|u^{+}\right\|^{2}, \quad \forall u \in X .
\end{gathered}
$$

The functional $\Phi$ defined by (14) can be rewritten as

$$
\Phi(u)=\frac{1}{2}\left(\left\|u^{+}\right\|^{2}-\left\|u^{-}\right\|^{2}\right)+\psi(u),
$$

where

$$
\psi(u)=\int_{\mathbb{R}^{N}} F(x, u) d x .
$$

The above variational setting for the functional (14) is standard. One can consult [5] or [6] for more details.

Let $\left\{e_{k}^{ \pm}\right\}$be the total orthonormal sequence in $X^{ \pm}$. Let $P$ : $X \rightarrow X^{-}, Q: X \rightarrow X^{+}$be the orthogonal projections. We define

$$
\left\||u \||=\max \left\{\|P u\|, \sum_{k=1}^{\infty} \frac{1}{2^{k+1}}\left|\left(Q u, e_{k}^{+}\right)\right|\right\}\right.
$$

on $X$. The topology generated by $\||\cdot|||$ is denoted by $\tau$, and all topological notation related to it will include this symbol.

Lemma 6. Suppose that (v) holds. Then

(a) $\max _{\mathbb{R}^{N}} V_{-} \geq \mu_{-1}$, where $\mu_{-1}$ is defined in $(\mathbf{v})$;

(b) for any $C>\mu_{-1}$, there exists $u_{0} \in X^{-}$with $\left\|u_{0}\right\|=1$ such that $C\left\|u_{0}\right\|_{L^{2}}>1$.

Proof. (a) We apply an indirect argument, and assume by contradiction that

$$
\max _{\mathbb{R}^{N}} V_{-}<\mu_{-1}
$$

From assumption $(\mathbf{v}),-\mu_{-1}$ is in the essential spectrum of the operator (with domain $D(L)=H^{2}\left(\mathbb{R}^{N}\right)$ ):

$$
L=-\Delta+V: L^{2}\left(\mathbb{R}^{N}\right) \longrightarrow L^{2}\left(\mathbb{R}^{N}\right) .
$$

Then, by Weyl's criterion (see, e.g., [25, Theorem VII.12] or [26, Theorem 7.2]), there exists a sequence $\left\{u_{n}\right\} \subset H^{2}\left(\mathbb{R}^{N}\right)$ with the properties that $\left\|u_{n}\right\|_{L^{2}}=1, \forall n$ and $\|-\Delta u_{n}+V u_{n}+$ $\mu_{-1} u_{n} \|_{L^{2}} \rightarrow 0$.

Since $\mu_{-1}>\max _{\mathbb{R}^{N}} V_{-}$, we deduce that $-V_{-}(x)+\mu_{-1}>0$ for all $x \in \mathbb{R}^{N}$. Together with the facts that $V$ is a continuous periodic function and $V=V_{+}-V_{-}$, this implies

$$
\inf _{x \in \mathbb{R}^{N}}\left(V(x)+\mu_{-1}\right)>0 .
$$

It follows that there exists a constant $C^{\prime}>0$ such that

$$
\int_{\mathbb{R}^{N}}\left(|\nabla u|^{2}+\left(V(x)+\mu_{-1}\right) u^{2}\right) d x \geq C^{\prime}\|u\|^{2}, \quad \forall u \in X .
$$

Note that

$$
\begin{aligned}
\int_{\mathbb{R}^{N}} & \left(-\Delta u_{n}+V(x) u_{n}+\mu_{-1} u_{n}\right) u_{n} d x \\
= & \int_{\mathbb{R}^{N}}\left(\left|\nabla u_{n}\right|^{2}+\left(V(x)+\mu_{-1}\right) u_{n}^{2}\right) d x .
\end{aligned}
$$

Together with (29) and the fact that $\|-\Delta u_{n}+V u_{n}+$ $\mu_{-1} u_{n} \|_{L^{2}} \rightarrow 0$ and $\left\|u_{n}\right\|_{L^{2}}=1$, this implies $\left\|u_{n}\right\| \rightarrow 0$. It contradicts $\left\|u_{n}\right\|_{L^{2}}=1, \forall n$. Therefore, $\max _{\mathbb{R}^{N}} V_{-} \geq \mu_{-1}$. 
(b) It suffices to prove that

$$
\mu_{-1}=C_{-}:=\inf \left\{\|u\|^{2} \mid u \in X^{-},\|u\|_{L^{2}}=1\right\} .
$$

From (21), we deduce that $\mu_{-1} \leq C_{-}$. From assumption (v), $-\mu_{-1}$ is in the essential spectrum of $L$. By Weyl's criterion, there exists $\left\{u_{n}\right\} \subset H^{2}\left(\mathbb{R}^{N}\right)$ such that $\left\|u_{n}\right\|_{L^{2}}=1$ and $\left\|-\Delta u_{n}+V u_{n}+\mu_{-1} u_{n}\right\|_{L^{2}} \rightarrow 0$. Multiplying $-\Delta u_{n}+V u_{n}+$ $\mu_{-1} u_{n}$ by $u_{n}^{+}$and then integrating it into $\mathbb{R}^{N}$, by (20) and (22), we get that

$$
\begin{aligned}
& \left(\mu_{1}+\mu_{-1}\right)\left\|u_{n}^{+}\right\|_{L^{2}}^{2} \\
& \quad \leq \int_{\mathbb{R}^{N}}\left(\left|\nabla u_{n}^{+}\right|^{2}+V(x)\left(u_{n}^{+}\right)^{2}+\mu_{-1}\left(u_{n}^{+}\right)^{2}\right) d x \\
& \quad=\int_{\mathbb{R}^{N}}\left(-\Delta u_{n}+V(x) u_{n}+\mu_{-1} u_{n}\right) u_{n}^{+} d x \longrightarrow 0 .
\end{aligned}
$$

It follows that $\left\|u_{n}^{-}\right\|_{L^{2}} \rightarrow 1$. Multiplying $-\Delta u_{n}+V u_{n}+\mu_{-1} u_{n}$ by $u_{n}^{-}$and then integrating it into $\mathbb{R}^{N}$, we get that

$$
\begin{aligned}
- & \left\|u_{n}^{-}\right\|^{2}+\mu_{-1}\left\|u_{n}^{-}\right\|_{L^{2}}^{2} \\
= & \int_{\mathbb{R}^{N}}\left(\left|\nabla u_{n}^{-}\right|^{2}+V(x)\left(u_{n}^{-}\right)^{2}+\mu_{-1}\left(u_{n}^{-}\right)^{2}\right) d x \\
= & \int_{\mathbb{R}^{N}}\left(-\Delta u_{n}+V u_{n}+\mu_{-1} u_{n}\right) u_{n}^{-} d x \longrightarrow 0 .
\end{aligned}
$$

It implies that $\mu_{-1} \geq C_{-}$. This together with $\mu_{-1} \leq C_{-}$implies $\mu_{-1}=C_{-}$.

Let $R>r>0$ and

$$
A:=\inf _{x \in \mathbb{R}^{N},|t| \geq D} \frac{f(x, t)}{t} .
$$

From assumption (5), we have $A>\max _{\mathbb{R}^{N}} V_{-}$. Together with the result $(\mathbf{a})$ of Lemma 6 , this implies that $(1 / 2)\left(A+\mu_{-1}\right)>$ $\mu_{-1}$. Choose

$$
\gamma \in\left(\mu_{-1}, \frac{\left(A+\mu_{-1}\right)}{2}\right) .
$$

Then by the result (b) of Lemma 6 , there exists $u_{0} \in X^{-}$with $\left\|u_{0}\right\|=1$ such that

$$
\gamma\left\|u_{0}\right\|_{L^{2}}>1
$$

Set

$$
\begin{gathered}
N=\left\{u \in X^{-} \mid\|u\|=r\right\}, \\
M=\left\{u \in X^{+} \oplus \mathbb{R}^{+} u_{0} \mid\|u\| \leq R\right\} .
\end{gathered}
$$

Then, $M$ is a submanifold of $X^{+} \oplus \mathbb{R}^{+} u_{0}$ with boundary

$$
\begin{aligned}
\partial M= & \left\{u \in X^{-} \mid\|u\| \leq R\right\} \\
& \cup\left\{u^{-}+t u_{0} \mid u^{-} \in X^{-}, t>0,\left\|u^{-}+t u_{0}\right\|=R\right\} .
\end{aligned}
$$

Definition 7. Let $\phi \in C^{1}(X ; \mathbb{R})$. A sequence $\left\{u_{n}\right\} \subset X$ is called a Palais-Smale sequence at level $c\left((P S)_{c}\right.$-sequence for short) for $\phi$, if $\phi\left(u_{n}\right) \rightarrow c$ and $\left\|\phi^{\prime}\left(u_{n}\right)\right\|_{X^{\prime}} \rightarrow 0$ as $n \rightarrow \infty$.

The following proposition is proved in [17] (see [17, Theorem 2.1]).

Proposition 8. Let $0<K<1$. The family of $C^{1}$-functional $\left\{H_{\lambda}\right\}$ has the form

$$
H_{\lambda}(u)=\lambda I(u)-J(u), \quad u \in X, \lambda \in[K, 1] .
$$

Assume

(a) $J(u) \geq 0, \forall u \in X$;

(b) $|I(u)|+J(u) \rightarrow+\infty$ as $\|u\| \rightarrow+\infty$;

(c) for all $\lambda \in[K, 1], H_{\lambda}$ is $\tau$-sequentially upper semicontinuous; that is, if $\left\|u_{n}-u\right\| \| \rightarrow 0$, then

$$
\limsup _{n \rightarrow \infty} H_{\lambda}\left(u_{n}\right) \leq H_{\lambda}(u) \text {, }
$$

and $H_{\lambda}^{\prime}$ is weakly sequentially continuous. Moreover, $H_{\lambda}$ maps bounded sets to bounded sets;

(d) there exist $u_{0} \in X^{-} \backslash\{0\}$ with $\left\|u_{0}\right\|=1$ and $R>r>0$ such that, for all $\lambda \in[K, 1]$,

$$
\inf _{N} H_{\lambda}>\sup _{\partial M} H_{\lambda} \text {. }
$$

Then there exists $E \subset[K, 1]$ such that the Lebesgue measure of $[K, 1] \backslash E$ is zero and, for every $\lambda \in E$, there exist $c_{\lambda}$ and $a$ bounded $(P S)_{c_{\lambda}}$-sequence for $H_{\lambda}$, where $c_{\lambda}$ satisfies

$$
\sup _{M} H_{\lambda} \geq c_{\lambda} \geq \inf _{N} H_{\lambda}
$$

For $0<K<1$ and $\lambda \in[K, 1]$, let

$$
\begin{aligned}
\Psi_{\lambda}(u)= & \frac{\lambda}{2} \int_{\mathbb{R}^{N}} V_{-}(x) u^{2} d x \\
& -\left(\frac{1}{2} \int_{\mathbb{R}^{N}}\left(|\nabla u|^{2}+V_{+}(x) u^{2}\right) d x+\psi(u)\right), \quad u \in X .
\end{aligned}
$$

Then

$$
\Psi_{1}=-\Phi
$$

and it is easy to verify that a critical point $u$ of $\Psi_{\lambda}$ is a weak solution of

$$
-\Delta u+V_{\lambda}(x) u+f(x, u)=0, \quad u \in X,
$$

where

$$
V_{\lambda}=V_{+}-\lambda V_{-}
$$

Lemma 9. Suppose that $(\mathbf{v})$ and $\left(\mathbf{f}_{1}\right)-\left(\mathbf{f}_{3}\right)$ hold. Then, there exist $0<K_{*}<1$ and $E \subset\left[K_{*}, 1\right]$ such that the Lebesgue measure of $\left[K_{*}, 1\right] \backslash E$ is zero and, for every $\lambda \in E$, there exist $c_{\lambda}$ and a bounded $(P S)_{c_{\lambda}}$-sequence for $\Psi_{\lambda}$, where $c_{\lambda}$ satisfies

$$
+\infty>\sup _{\lambda \in E} c_{\lambda} \geq \inf _{\lambda \in E} c_{\lambda}>0 .
$$


Proof. For $u \in X$, let

$$
\begin{gathered}
I(u)=\frac{1}{2} \int_{\mathbb{R}^{N}} V_{-}(x) u^{2} d x \\
J(u)=\frac{1}{2} \int_{\mathbb{R}^{N}}\left(|\nabla u|^{2}+V_{+}(x) u^{2}\right) d x+\psi(u) .
\end{gathered}
$$

Then, $I$ and $J$ satisfy assumptions (a) and (b) in Proposition 8, and, by $(43), \Psi_{\lambda}(u)=\lambda I(u)-J(u)$.

From (43) and (20), for any $u \in X$ and $\lambda \in[K, 1]$, we have

$$
\begin{aligned}
\Psi_{\lambda}(u)= & \frac{\lambda-1}{2} \int_{\mathbb{R}^{N}} V_{-}(x) u^{2} d x \\
& -\left(\frac{1}{2} \int_{\mathbb{R}^{N}}\left(|\nabla u|^{2}+V(x) u^{2}\right) d x+\int_{\mathbb{R}^{N}} F(x, u) d x\right) \\
= & \frac{1}{2}\left\|u^{-}\right\|^{2}-\frac{1}{2}\left\|u^{+}\right\|^{2} \\
& -\frac{1-\lambda}{2} \int_{\mathbb{R}^{N}} V_{-}(x) u^{2} d x-\int_{\mathbb{R}^{N}} F(x, u) d x .
\end{aligned}
$$

Let $u_{*} \in X$ and $\left\{u_{n}\right\} \subset X$ be such that $\left\|u_{n}-u_{*}\right\| \| \rightarrow 0$. It follows that $u_{n}^{-} \rightarrow u_{*}^{-}, u_{n}^{+} \rightarrow u_{*}^{+}$, and $u_{n} \rightarrow u_{*}$. In addition, up to a subsequence, we can assume that $u_{n} \rightarrow u_{*}$ a.e. in $\mathbb{R}^{N}$. Then, we have

$$
\begin{gathered}
\left\|u_{n}^{-}\right\|^{2} \longrightarrow\left\|u_{*}^{-}\right\|^{2}, \\
\liminf _{n \rightarrow \infty} \int_{\mathbb{R}^{N}} V_{-}(x) u_{n}^{2} d x \\
\geq \int_{\mathbb{R}^{N}} V_{-}(x) u_{*}^{2} d x \quad \text { (by Fatou's lemma), } \\
\liminf _{n \rightarrow \infty}\left\|u_{n}^{+}\right\|^{2} \geq\left\|u_{*}^{+}\right\|^{2} .
\end{gathered}
$$

By Remark $1, F(x, t) \geq 0$ for all $x$ and $t$. This together with Fatou's lemma implies

$$
\liminf _{n \rightarrow \infty} \int_{\mathbb{R}^{N}} F\left(x, u_{n}\right) d x \geq \int_{\mathbb{R}^{N}} F\left(x, u_{*}\right) d x
$$

Then, by (49), we obtain

$$
\limsup _{n \rightarrow \infty} \Psi_{\lambda}\left(u_{n}\right) \leq \Psi_{\lambda}\left(u_{*}\right)
$$

This implies that $\Psi_{\lambda}$ is $\tau$-sequentially upper semicontinuous.

If $u_{n} \rightarrow u_{*}$ in $X$, then, for any fixed $\varphi \in X$, as $n \rightarrow \infty$,

$$
\begin{aligned}
& \left\langle-\Psi_{\lambda}^{\prime}\left(u_{n}\right), \varphi\right\rangle \\
& =\int_{\mathbb{R}^{N}}\left(\nabla u_{n} \nabla \varphi+V_{\lambda} u_{n} \varphi\right) d x+\int_{\mathbb{R}^{N}} f\left(x, u_{n}\right) \varphi d x \\
& \longrightarrow \int_{\mathbb{R}^{N}}\left(\nabla u_{*} \nabla \varphi+V_{\lambda} u_{*} \varphi\right) d x+\int_{\mathbb{R}^{N}} f\left(x, u_{*}\right) \varphi d x \\
& =\left\langle-\Psi_{\lambda}^{\prime}\left(u_{*}\right), \varphi\right\rangle .
\end{aligned}
$$

This implies that $\Psi_{\lambda}^{\prime}$ is weakly sequentially continuous. Moreover, it is easy to see that $\Psi_{\lambda}$ maps bounded sets to bounded sets. Therefore, $\Psi_{\lambda}$ satisfies assumption (c) in Proposition 8.

Finally, we will verify assumption (d) in Proposition 8 for $\Psi_{\lambda}$.

From assumption $\left(\mathbf{f}_{\mathbf{1}}\right)$ and $f(x, t) / t \rightarrow 0$ as $t \rightarrow 0$ uniformly for $x \in \mathbb{R}^{N}$, we deduce that, for any $\epsilon>0$, there exists $C_{\epsilon}>0$ such that

$$
F(x, t) \leq \epsilon t^{2}+C_{\epsilon}|t|^{p}, \quad \forall(x, t) \in \mathbb{R}^{N} \times \mathbb{R} .
$$

From (49) and (55), we have, for $u \in N$,

$$
\begin{aligned}
\Psi_{\lambda}(u) \geq & \frac{1}{2}\|u\|^{2}-\frac{1-\lambda}{2} \int_{\mathbb{R}^{N}} V_{-}(x) u^{2} d x \\
& -\epsilon \int_{\mathbb{R}^{N}} u^{2} d x-C_{\epsilon} \int_{\mathbb{R}^{N}}|u|^{p} d x .
\end{aligned}
$$

Then by the Sobolev inequality $\|u\|_{L^{p}\left(\mathbb{R}^{N}\right)} \leq C\|u\|$ and $\|u\|_{L^{2}} \leq$ $C\|u\|$ (by (21) and (22)), we deduce that there exists a constant $C>0$ such that

$$
\begin{aligned}
\Psi_{\lambda}(u) \geq & \frac{1}{2}\|u\|^{2}-C(1-\lambda) \max _{\mathbb{R}^{N}} V_{-}(x)\|u\|^{2} \\
& -\epsilon C\|u\|^{2}-C C_{\epsilon}\|u\|^{p} .
\end{aligned}
$$

Choose $0<K_{*}<1$ and $\epsilon>0$ such that $C\left(1-K_{*}\right)$ $\max _{\mathbb{R}^{N}} V_{-}(x)<1 / 4$ and $C_{\epsilon}=1 / 8$. Then, for every $\lambda \in\left[K_{*}, 1\right]$, we have

$$
\Psi_{\lambda}(u) \geq \frac{1}{8}\|u\|^{2}-C C_{\epsilon}\|u\|^{p}
$$

Let $r>0$ be such that $r^{p-2} C C_{\epsilon}=1 / 16$ and $\beta=r^{2} / 16$. Then, from (58), we deduce that, for $N=\left\{u \in X^{-} \mid\|u\|=r\right\}$,

$$
\inf _{N} \Psi_{\lambda} \geq \beta, \quad \forall \lambda \in\left[K_{*}, 1\right]
$$

We will prove that $\sup _{K_{*} \leq \lambda \leq 1} \Psi_{\lambda}(u) \rightarrow-\infty$ as $\|u\| \rightarrow \infty$ and $u \in X^{+} \oplus \mathbb{R}^{+} u_{0}$. Arguing indirectly, assume that, for some sequences $\lambda_{n} \in\left[K_{*}, 1\right]$ and $u_{n} \in X^{+} \oplus \mathbb{R}^{+} u_{0}$ with $\left\|u_{n}\right\| \rightarrow+\infty$, there is $\mathscr{L}>0$ such that $\Psi_{\lambda_{n}}\left(u_{n}\right) \geq-\mathscr{L}$ for all $n$. Then, setting $w_{n}=u_{n} /\left\|u_{n}\right\|$, we have $\left\|w_{n}\right\|=1$, and, up to a subsequence, $w_{n} \rightarrow w, w_{n}^{-} \rightarrow w^{-} \in X^{-}$and $w_{n}^{+} \rightarrow w^{+} \in X^{+}$.

First, we consider the case $w \neq 0$. Dividing both sides of (49) by $\left\|u_{n}\right\|^{2}$, we get that

$$
\begin{aligned}
-\frac{\mathscr{L}}{\left\|u_{n}\right\|^{2}} \leq & \frac{\Psi_{\lambda_{n}}\left(u_{n}\right)}{\left\|u_{n}\right\|^{2}} \\
= & \frac{1}{2}\left\|w_{n}^{-}\right\|^{2}-\frac{1}{2}\left\|w_{n}^{+}\right\|^{2} \\
& -\frac{1-\lambda_{n}}{2} \int_{\mathbb{R}^{N}} V_{-}(x) w_{n}^{2} d x-\int_{\mathbb{R}^{N}} \frac{F\left(x, u_{n}\right)}{\left\|u_{n}\right\|^{2}} d x
\end{aligned}
$$

From (5) and the result (a) of Lemma 6, we deduce that

$$
\liminf _{|t| \rightarrow \infty} \frac{F(x, t)}{t^{2}} \geq \frac{A}{2}>\frac{1}{2} \max _{\mathbb{R}^{N}} V_{-} \geq \frac{1}{2} \mu_{-1},
$$


where $A$ is defined by (34). Note that, for $x \in\left\{x \in \mathbb{R}^{N}\right.$ | $w \neq 0\}$, we have $\left|u_{n}(x)\right| \rightarrow+\infty$. This implies that, when $n$ is large enough,

$$
\int_{\left\{x \in \mathbb{R}^{N} \mid w \neq 0\right\}} \frac{F\left(x, u_{n}\right)}{u_{n}^{2}} w_{n}^{2} d x \geq \frac{A+\mu_{-1}}{4} \int_{\left\{x \in \mathbb{R}^{N} \mid w \neq 0\right\}} w_{n}^{2} d x .
$$

By (10), we have, when $n$ is large enough,

$$
\begin{aligned}
\int_{\mathbb{R}^{N}} \frac{F\left(x, u_{n}\right)}{\left\|u_{n}\right\|^{2}} d x & =\int_{\mathbb{R}^{N}} \frac{F\left(x, u_{n}\right)}{u_{n}^{2}} w_{n}^{2} d x \\
& \geq \int_{\left\{x \in \mathbb{R}^{N} \mid w \neq 0\right\}} \frac{F\left(x, u_{n}\right)}{u_{n}^{2}} w_{n}^{2} d x .
\end{aligned}
$$

Combining the above two inequalities yields

$$
\begin{aligned}
& \liminf _{n \rightarrow \infty}\left(\frac{1}{2}\left\|w_{n}^{-}\right\|^{2}-\frac{1}{2}\left\|w_{n}^{+}\right\|^{2}\right. \\
& \left.\quad-\frac{1-\lambda_{n}}{2} \int_{\mathbb{R}^{N}} V_{-}(x) w_{n}^{2} d x-\int_{\mathbb{R}^{N}} \frac{F\left(x, u_{n}\right)}{\left\|u_{n}\right\|^{2}} d x\right) \\
& \leq \liminf _{n \rightarrow \infty}\left(\frac{1}{2}\left\|w_{n}^{-}\right\|^{2}-\frac{1}{2}\left\|w_{n}^{+}\right\|^{2}\right. \\
& \left.\quad-\frac{A+\mu_{-1}}{4} \int_{\left\{x \in \mathbb{R}^{N} \mid w \neq 0\right\}} w_{n}^{2} d x\right) \\
& \leq \frac{1}{2}\left\|w^{-}\right\|^{2}-\frac{1}{2}\left\|w^{+}\right\|^{2}-\frac{A+\mu_{-1}}{4} \int_{\mathbb{R}^{N}} w^{2} d x \\
& \leq \frac{1}{2}\left\|w^{-}\right\|^{2}-\frac{1}{2}\left\|w^{+}\right\|^{2}-\frac{A+\mu_{-1}}{4}\left\|w^{-}\right\|_{L^{2}}^{2} .
\end{aligned}
$$

We used the inequalities

$$
\begin{gathered}
\lim _{n \rightarrow \infty}\left\|w_{n}^{-}\right\|^{2}=\left\|w^{-}\right\|^{2}, \\
\liminf _{n \rightarrow \infty}\left\|w_{n}^{+}\right\|^{2} \geq\left\|w^{+}\right\|^{2}, \\
\liminf _{n \rightarrow \infty} \int_{\left\{x \in \mathbb{R}^{N} \mid w \neq 0\right\}} w_{n}^{2} d x \geq \int_{\mathbb{R}^{N}} w^{2} d x
\end{gathered}
$$

in the second inequality of (64).

Since $w^{-}=t u_{0}$ for some $t \in \mathbb{R}$, by (36), we get that

$$
\frac{A+\mu_{-1}}{4}\left\|w^{-}\right\|_{L^{2}}^{2} \geq \frac{A+\mu_{-1}}{4 \gamma}\left\|w^{-}\right\|^{2} .
$$

Note that, by the choice of $\gamma$ (see (35)), we have $((A+$ $\left.\left.\mu_{-1}\right) / 4 \gamma\right)>1 / 2$. Then by (64) and the fact that $w \neq 0$, we have that

$$
\begin{aligned}
& \liminf _{n \rightarrow \infty}\left(\frac{1}{2}\left\|w_{n}^{-}\right\|^{2}-\frac{1}{2}\left\|w_{n}^{+}\right\|^{2}\right. \\
& \left.\quad-\frac{1-\lambda_{n}}{2} \int_{\mathbb{R}^{N}} V_{-}(x) w_{n}^{2} d x-\int_{\mathbb{R}^{N}} \frac{F\left(x, u_{n}\right)}{\left\|u_{n}\right\|^{2}} d x\right) \\
& \leq-\left(\frac{A+\mu_{-1}}{4 \gamma}-\frac{1}{2}\right)\left\|w^{-}\right\|^{2}-\frac{1}{2}\left\|w^{+}\right\|^{2}<0
\end{aligned}
$$

It contradicts (60), since $-\mathscr{L} /\left\|u_{n}\right\|^{2} \rightarrow 0$ as $n \rightarrow \infty$.

Second, we consider the case $w=0$. In this case, $\lim _{n \rightarrow \infty}\left\|w_{n}^{-}\right\|=0$. It follows that

$$
\liminf _{n \rightarrow \infty}\left\|w_{n}^{+}\right\| \geq 1,
$$

since $\left\|w_{n}\right\|=1$ and $w_{n}=w_{n}^{+}+w_{n}^{-}$. Therefore, the right hand side of $(60)$ is less than $-1 / 4$ when $n$ is large enough. However, as $n \rightarrow \infty$, the left hand side of (60) converges to zero. It induces a contradiction.

Therefore, there exists $R>r$ such that

$$
\sup _{\lambda \in\left[K_{*}, 1\right]} \sup _{\partial M} \Psi_{\lambda} \leq 0
$$

This implies that $\Psi_{\lambda}$ satisfies assumption (d) in Proposition 8 if $\lambda \in\left[K_{*}, 1\right]$. Finally, it is easy to see that

$$
\sup _{\lambda \in\left[K_{*}, 1\right]} \sup _{M} \Psi_{\lambda}<+\infty
$$

Then, the results of this lemma follow immediately from Proposition 8.

Lemma 10. Suppose that $(\mathbf{v})$ and $\left(\mathbf{f}_{\mathbf{1}}\right)-\left(\mathbf{f}_{\mathbf{3}}\right)$ are satisfied. Let $\lambda \epsilon$ $\left[K_{*}, 1\right]$ be fixed, where $K_{*}$ is the constant in Lemma 9. If $\left\{v_{n}\right\}$ is a bounded $(P S)_{c}$-sequence for $\Psi_{\lambda}$ with $c \neq 0$, then, for every $n \in \mathbb{N}$, there exists $a_{n} \in \mathbb{Z}^{N}$ such that, up to a subsequence, $u_{n}:=v_{n}\left(\cdot+a_{n}\right)$ satisfies

$$
u_{n} \rightarrow u_{\lambda} \neq 0, \quad \Psi_{\lambda}\left(u_{\lambda}\right) \leq c, \quad \Psi_{\lambda}^{\prime}\left(u_{\lambda}\right)=0 .
$$

Proof. The proof of this lemma is inspired by the proof of Lemma 3.7 in [19]. Because $\left\{v_{n}\right\}$ is a bounded sequence in $X$, up to a subsequence, either

(a) $\lim _{n \rightarrow \infty} \sup _{y \in \mathbb{R}^{N}} \int_{B_{1}(y)}\left|v_{n}\right|^{2} d x=0$ or

(b) there exist $\varrho>0$ and $a_{n} \in \mathbb{Z}^{N}$ such that $\int_{B_{1}\left(a_{n}\right)}\left|v_{n}\right|^{2} d x \geq \varrho$.

If (a) occurs, using the Lions lemma (see, e.g., [21, Lemma 1.21]), a similar argument as for the proof of [19, Lemma 3.6] shows that

$$
\lim _{n \rightarrow \infty} \int_{\mathbb{R}^{N}} F\left(x, v_{n}\right) d x=0, \quad \lim _{n \rightarrow \infty} \int_{\mathbb{R}^{N}} f\left(x, v_{n}\right) v_{n}^{ \pm} d x=0 .
$$


It follows that

$$
\lim _{n \rightarrow \infty} \int_{\mathbb{R}^{N}}\left(2 F\left(x, v_{n}\right)-f\left(x, v_{n}\right) v_{n}\right) d x=0 .
$$

On the other hand, as $\left\{v_{n}\right\}$ is a $(P S)_{c}$-sequence of $\Psi_{\lambda}$, we have $\left\langle\Psi_{\lambda}^{\prime}\left(v_{n}\right), v_{n}\right\rangle \rightarrow 0$ and $\Psi_{\lambda}\left(v_{n}\right) \rightarrow c \neq 0$. It follows that

$$
\begin{aligned}
& \int_{\mathbb{R}^{N}}\left(f\left(x, v_{n}\right) v_{n}-2 F\left(x, v_{n}\right)\right) d x \\
& \quad=2 \Psi_{\lambda}\left(v_{n}\right)-\left\langle\Psi_{\lambda}^{\prime}\left(v_{n}\right), v_{n}\right\rangle \longrightarrow 2 c \neq 0, \quad n \longrightarrow \infty
\end{aligned}
$$

This contradicts (73). Therefore, case (a) cannot occur.

If case (b) occurs, let $u_{n}=v_{n}\left(\cdot+a_{n}\right)$. For every $n$,

$$
\int_{B_{1}(0)}\left|u_{n}\right|^{2} d x \geq \varrho .
$$

Because $V$ and $F(x, t)$ are 1-periodic in every $x_{j},\left\{u_{n}\right\}$ is still bounded in $X$,

$$
\lim _{n \rightarrow \infty} \Psi_{\lambda}\left(u_{n}\right) \leq c, \quad \Psi_{\lambda}^{\prime}\left(u_{n}\right) \rightarrow 0, \quad n \longrightarrow \infty .
$$

Up to a subsequence, we assume that $u_{n} \rightarrow u_{\lambda}$ in $X$ as $n \rightarrow \infty$. Since $u_{n} \rightarrow u_{\lambda}$ in $L_{\text {loc }}^{2}\left(\mathbb{R}^{N}\right)$, it follows from (75) that $u_{\lambda} \neq 0$. Recall that $\Psi_{\lambda}^{\prime}\left(u_{n}\right)$ is weakly sequentially continuous. Therefore, $\Psi_{\lambda}^{\prime}\left(u_{n}\right) \rightarrow \Psi_{\lambda}^{\prime}\left(u_{\lambda}\right)$ and, by $(76), \Psi_{\lambda}^{\prime}\left(u_{\lambda}\right)=0$.

Finally, by $\left(\mathbf{f}_{\mathbf{3}}\right)$ and Fatou's lemma

$$
\begin{aligned}
c & =\lim _{n \rightarrow \infty}\left(\Psi_{\lambda}\left(u_{n}\right)-\frac{1}{2}\left\langle\Psi_{\lambda}^{\prime}\left(u_{n}\right), u_{n}\right\rangle\right) \\
& =\lim _{n \rightarrow \infty} \int_{\mathbb{R}^{N}} \widetilde{F}\left(x, u_{n}\right) \geq \int_{\mathbb{R}^{N}} \widetilde{F}\left(x, u_{\lambda}\right)=\Psi_{\lambda}\left(u_{\lambda}\right) .
\end{aligned}
$$

Lemma 11. There exist $0<K_{* *}<1$ and $\eta>0$ such that, for any $\lambda \in\left[K_{* *}, 1\right]$, if $u \neq 0$ satisfies $\Psi_{\lambda}^{\prime}(u)=0$, then $\|u\| \geq \eta$.

Proof. We adapt the arguments of Yang [23, p. 2626] and Liu [12, Lemma 2.2]. Note that, by $\left(\mathbf{f}_{1}\right)$ and $\left(\mathbf{f}_{2}\right)$, for any $\epsilon>0$, there exists $C_{\epsilon}>0$ such that

$$
|f(x, t)| \leq \epsilon|t|+C_{\epsilon}|t|^{p-1} .
$$

Let $u \neq 0$ be a critical point of $\Psi_{\lambda}$. Then $u$ is a solution of

$$
-\Delta u+V_{\lambda} u+f(x, u)=0, \quad u \in X
$$

Multiplying both sides of this equation by $u^{ \pm}$, respectively, and then integrating into $\mathbb{R}^{N}$, we get that

$$
\begin{aligned}
0= & \pm\left\|u^{ \pm}\right\|^{2}+(1-\lambda) \int_{\mathbb{R}^{N}} V_{-}(x) u_{n} u^{ \pm} d x \\
& +\int_{\mathbb{R}^{N}} f(x, u) u^{ \pm} d x .
\end{aligned}
$$

It follows that

$$
\begin{aligned}
\left\|u^{ \pm}\right\|^{2}= & \mp(1-\lambda) \int_{\mathbb{R}^{N}} V_{-}(x) u u^{ \pm} d x \mp \int_{\mathbb{R}^{N}} f(x, u) u^{ \pm} d x \\
\leq & (1-\lambda) \sup _{\mathbb{R}^{N}} V_{-} \int_{\mathbb{R}^{N}}|u| \cdot\left|u^{ \pm}\right| d x \\
& +\epsilon \int_{\mathbb{R}^{N}}|u| \cdot\left|u^{ \pm}\right| d x+C_{\epsilon} \int_{\mathbb{R}^{N}}|u|^{p-1}\left|u^{ \pm}\right| d x \\
\leq & C_{1}((1-\lambda)+\epsilon)\|u\| \cdot\left\|u^{ \pm}\right\|+C_{2}\|u\|^{p-1}\left\|u^{ \pm}\right\|
\end{aligned}
$$

where $C_{1}$ and $C_{2}$ are positive constants related to the Sobolev inequalities and $\sup _{\mathbb{R}^{N}} V_{-}$. From the above two inequalities, we obtain

$$
\|u\|^{2}=\left\|u^{+}\right\|^{2}+\left\|u^{-}\right\|^{2} \leq 2 C_{1}((1-\lambda)+\epsilon)\|u\|^{2}+2 C_{2}\|u\|^{p} .
$$

Because $p>2$, this implies that $\|u\| \geq \eta$ for some $\eta>0$ if $\epsilon>0$ and $1-K_{* *}>0$ are small enough and $\lambda \in\left[K_{* *}, 1\right]$. The desired result follows.

Let $K=\max \left\{K_{*}, K_{* *}\right\}$, where $K_{*}$ and $K_{* *}$ are the constants that appeared in Lemmas 9 and 11, respectively. Combining Lemmas 9-11, we obtain the following lemma.

Lemma 12. Suppose $(\mathbf{v})$ and $\left(\mathbf{f}_{\mathbf{1}}\right)-\left(\mathbf{f}_{\mathbf{3}}\right)$ are satisfied. Then, there exist $\eta>0,\left\{\lambda_{n}\right\} \subset[K, 1]$, and $\left\{u_{n}\right\} \subset X$ such that $\lambda_{n} \rightarrow 1$,

$$
\sup _{n} \Psi_{\lambda_{n}}\left(u_{n}\right)<+\infty, \quad\left\|u_{n}\right\| \geq \eta, \quad \Psi_{\lambda_{n}}^{\prime}\left(u_{n}\right)=0
$$

\section{A Priori Bound of Approximate Solutions and Proof of the Main Theorem}

In this section, we give a priori bound for the sequence of approximate solutions $\left\{u_{n}\right\}$ obtained in Lemma 12. We then give the proofs of Theorem 3 .

Lemma 13. Suppose $(\mathbf{v})$ and $\left(\mathbf{f}_{\mathbf{1}}\right)-\left(\mathbf{f}_{\mathbf{3}}\right)$ are satisfied. Let $\left\{u_{n}\right\}$ be the sequence obtained in Lemma 12. Then, $\left\{u_{n}\right\} \subset L^{\infty}\left(\mathbb{R}^{N}\right)$ and

$$
\sup _{n}\left\|u_{n}\right\|_{L^{\infty}\left(\mathbb{R}^{N}\right)} \leq D
$$

Proof. From $\Psi_{\lambda_{n}}^{\prime}\left(u_{n}\right)=0$, we deduce that $u_{n}$ is a weak solution of (45) with $\lambda=\lambda_{n}$; that is,

$$
-\Delta u_{n}+V_{\lambda_{n}}(x) u_{n}+f\left(x, u_{n}\right)=0 \quad \text { in } \mathbb{R}^{N} .
$$

By assumption $\left(\mathbf{f}_{\mathbf{1}}\right)$ and the bootstrap argument of elliptic equations, we deduce that $u_{n} \in L^{\infty}\left(\mathbb{R}^{N}\right)$.

Multiplying both sides of $(85)$ by $v_{n}=\left(u_{n}-D\right)^{+}:=$ $\max \left\{u_{n}-D, 0\right\}$ and integrating into $\mathbb{R}^{N}$, we get that

$$
\int_{\mathbb{R}^{N}}\left|\nabla v_{n}\right|^{2} d x+\int_{u_{n} \geq D}\left(V_{\lambda_{n}}(x) u_{n}+f\left(x, u_{n}\right)\right) v_{n} d x=0 .
$$


Recall that $V_{\lambda_{n}}=V_{+}-\lambda_{n} V_{-}$and $\lambda_{n} \leq 1$. Then by (5), we get that

$$
\begin{aligned}
& \int_{u_{n} \geq D}\left(V_{\lambda_{n}}(x) u_{n}+f\left(x, u_{n}\right)\right) v_{n} d x \\
& =\int_{u_{n} \geq D}\left(V_{\lambda_{n}}(x)+\frac{f\left(x, u_{n}\right)}{u_{n}}\right) u_{n} v_{n} d x \geq 0 .
\end{aligned}
$$

This together with (86) yields $v_{n}=0$. It follows that $u_{n}(x) \leq D$ on $\mathbb{R}^{N}$.

Similarly, multiplying both sides of (85) by $w_{n}=\left(u_{n}+\right.$ $D)^{-}:=\max \left\{-\left(u_{n}+D\right), 0\right\}$ and integrating into $\mathbb{R}^{N}$, we can get that $u_{n} \geq-D$ on $\mathbb{R}^{N}$. Therefore, for all $n,\left\|u_{n}\right\|_{L^{\infty}\left(\mathbb{R}^{N}\right)} \leq D$.

Lemma 14. Suppose that $(\mathbf{v}),\left(\mathbf{f}_{\mathbf{1}}\right),\left(\mathbf{f}_{\mathbf{2}}\right),\left(\mathbf{f}_{\mathbf{3}}\right)$, and $\left(\mathbf{f}_{\mathbf{4}}\right)$ are satisfied. Let $\left\{u_{n}\right\}$ be the sequence obtained in Lemma 12. Then

$$
0<\inf _{n}\left\|u_{n}\right\| \leq \sup _{n}\left\|u_{n}\right\|<+\infty .
$$

Proof. As $\Psi_{\lambda_{n}}^{\prime}\left(u_{n}\right)=0$ and $u_{n} \neq 0$, Lemma 11 implies that $\inf _{n}\left\|u_{n}\right\|>0$.

To prove $\sup _{n}\left\|u_{n}\right\|<+\infty$, we apply an indirect argument and assume by contradiction that $\left\|u_{n}\right\| \rightarrow+\infty$.

Since $\Psi_{\lambda_{n}}^{\prime}\left(u_{n}\right)=0$, by $(81)$, we get that

$$
\begin{aligned}
\left\|u_{n}^{ \pm}\right\|^{2} & =\mp\left(1-\lambda_{n}\right) \int_{\mathbb{R}^{N}} V_{-}(x) u_{n} u_{n}^{ \pm} d x \mp \int_{\mathbb{R}^{N}} f\left(x, u_{n}\right) u_{n}^{ \pm} d x \\
& =\mp \int_{\mathbb{R}^{N}} f\left(x, u_{n}\right) u_{n}^{ \pm} d x+\left(1-\lambda_{n}\right) O\left(\left\|u_{n}\right\|^{2}\right) .
\end{aligned}
$$

It follows that

$$
\begin{aligned}
\left\|u_{n}\right\|^{2} & +\int_{\mathbb{R}^{N}} f\left(x, u_{n}\right)\left(u_{n}^{+}-u_{n}^{-}\right) d x \\
= & \left\|u_{n}^{+}\right\|^{2}+\left\|u_{n}^{-}\right\|^{2} \\
& +\int_{\mathbb{R}^{N}} f\left(x, u_{n}\right)\left(u_{n}^{+}-u_{n}^{-}\right) d x \\
= & \left(1-\lambda_{n}\right) O\left(\left\|u_{n}\right\|^{2}\right) .
\end{aligned}
$$

Set $w_{n}=u_{n} /\left\|u_{n}\right\|$. Then, by (90),

$$
\begin{gathered}
\left\|u_{n}\right\|^{2}\left(1+\int_{\mathbb{R}^{N}} \frac{f\left(x, u_{n}\right)}{u_{n}}\left(w_{n}^{+}-w_{n}^{-}\right) w_{n} d x\right) \\
=\left(1-\lambda_{n}\right) O\left(\left\|u_{n}\right\|^{2}\right) .
\end{gathered}
$$

Then, by $\lambda_{n} \rightarrow 1$ as $n \rightarrow \infty$, we have that

$$
\int_{\mathbb{R}^{N}} \frac{f\left(x, u_{n}\right)}{u_{n}}\left(w_{n}^{+}-w_{n}^{-}\right) w_{n} d x \longrightarrow-1, \quad n \longrightarrow \infty .
$$

From Lemma 12,

$$
C_{0}:=\sup _{n} \Psi_{\lambda_{n}}\left(u_{n}\right)<+\infty .
$$

Then, by $\Psi_{\lambda_{n}}^{\prime}\left(u_{n}\right)=0$, we obtain

$$
2 C_{0} \geq 2 \Psi_{\lambda_{n}}\left(u_{n}\right)-\left\langle\Psi_{\lambda_{n}}^{\prime}\left(u_{n}\right), u_{n}\right\rangle=2 \int_{\mathbb{R}^{N}} \widetilde{F}\left(x, u_{n}\right) d x .
$$

From $\left(\mathbf{f}_{3}\right)$, we have

$$
2 C_{0} \geq 2 \int_{\mathbb{R}^{N}} \widetilde{F}\left(x, u_{n}\right) d x \geq 2 \int_{\left\{x|D \geq| u_{n}(x) \mid \geq \kappa\right\}} \widetilde{F}\left(x, u_{n}\right) d x,
$$

where $\kappa$ is the constant in $\left(\mathbf{f}_{\mathbf{4}}\right)$. As the continuous function $\widetilde{F}$ is 1 -periodic in every $x_{j}$ variable, we deduce from (8) that there exists a constant $C^{\prime}>0$ such that

$$
\begin{aligned}
& \widetilde{F}(x, t) \geq C^{\prime} t^{2}, \\
& \quad \text { for every }(x, t) \in \mathbb{R}^{N} \times \mathbb{R} \text { with } \kappa \leq|t| \leq D .
\end{aligned}
$$

Combining (95) and (96) leads to

$$
C_{0} \geq C^{\prime} \int_{\left\{x|D \geq| u_{n}(x) \mid \geq \kappa\right\}} u_{n}^{2} d x
$$

Dividing both sides of this inequality by $\left\|u_{n}\right\|^{2}$ and sending $n \rightarrow \infty$, we obtain

$$
\lim _{n \rightarrow \infty} \int_{\left\{x|D \geq| u_{n}(x) \mid \geq \kappa\right\}} w_{n}^{2} d x=0 .
$$

From (7), (21), and (22), we have that

$$
\begin{gathered}
\int_{\left\{x \| u_{n}(x) \mid<\kappa\right\}}\left|\frac{f\left(x, u_{n}\right)}{u_{n}}\left(w_{n}^{+}-w_{n}^{-}\right) w_{n}\right| d x \\
\leq v \int_{\left\{x \| u_{n}(x) \mid<\kappa\right\}}\left|\left(w_{n}^{+}-w_{n}^{-}\right) w_{n}\right| d x \\
\leq v \int_{\mathbb{R}^{N}}\left|\left(w_{n}^{+}-w_{n}^{-}\right) w_{n}\right| d x \\
\leq v\left\|w_{n}\right\|_{L^{2}}^{2} \leq \frac{v}{\mu_{0}}\left\|w_{n}\right\|^{2}=\frac{v}{\mu_{0}}<1,
\end{gathered}
$$

where $\mu_{0}$ is the constant defined in $(\mathbf{v})$.

Since $f \in C\left(\mathbb{R}^{N} \times \mathbb{R}\right)$ and $\lim _{t \rightarrow 0} f(x, t) / t=0$, we deduce that there exists $C>0$ such that, for every $(x, t) \in \mathbb{R}^{N} \times \mathbb{R}$ with $|t| \leq D$,

$$
|f(x, t)| \leq C|t| .
$$

This together with (98) gives

$$
\begin{aligned}
& \int_{\left\{x|D \geq| u_{n}(x) \mid \geq \kappa\right\}}\left|\frac{f\left(x, u_{n}\right)}{u_{n}}\left(w_{n}^{+}-w_{n}^{-}\right) w_{n}\right| d x \\
& \leq C \int_{\left\{x|D \geq| u_{n}(x) \mid \geq \kappa\right\}}\left|\left(w_{n}^{+}-w_{n}^{-}\right) w_{n}\right| d x \\
& \leq C\left\|w_{n}^{+}-w_{n}^{-}\right\|_{L^{2}}\left(\int_{\left\{x|D \geq| u_{n}(x) \mid \geq \kappa\right\}} w_{n}^{2} d x\right)^{1 / 2} \\
& \quad \leq 2 C\left\|w_{n}\right\|_{L^{2}}\left(\int_{\left\{x|D \geq| u_{n}(x) \mid \geq \kappa\right\}} w_{n}^{2} d x\right)^{1 / 2} \longrightarrow 0, \quad n \rightarrow \infty .
\end{aligned}
$$


Combining (99) and (101) yields

$$
\begin{aligned}
& \limsup _{n \rightarrow \infty} \int_{\mathbb{R}^{N}}\left|\frac{f\left(x, u_{n}\right)}{u_{n}}\left(w_{n}^{+}-w_{n}^{-}\right) w_{n}\right| d x \\
& \leq \underset{n \rightarrow \infty}{\limsup } \int_{\left\{x|| u_{n}(x) \mid<\kappa\right\}}\left|\frac{f\left(x, u_{n}\right)}{u_{n}}\left(w_{n}^{+}-w_{n}^{-}\right) w_{n}\right| d x \\
& \quad+\limsup _{n \rightarrow \infty} \int_{\left\{x|D \geq| u_{n}(x) \mid \geq \kappa\right\}}\left|\frac{f\left(x, u_{n}\right)}{u_{n}}\left(w_{n}^{+}-w_{n}^{-}\right) w_{n}\right| d x<1 .
\end{aligned}
$$

This contradicts (92). Therefore, $\left\{u_{n}\right\}$ is bounded in $X$.

Proof of Theorem 3. Let $\left\{u_{n}\right\}$ be the sequence obtained in Lemma 12. From Lemma $14,\left\{u_{n}\right\}$ is bounded in $X$. Therefore, up to a subsequence, either

(a) $\lim _{n \rightarrow \infty} \sup _{y \in \mathbb{R}^{N}} \int_{B_{1}(y)}\left|u_{n}\right|^{2} d x=0$ or

(b) there exist $\varrho>0$ and $y_{n} \in \mathbb{Z}^{N}$ such that $\int_{B_{1}\left(y_{n}\right)}\left|u_{n}\right|^{2} d x \geq \varrho$.

According to (72), if case (a) occurs,

$$
\lim _{n \rightarrow \infty} \int_{\mathbb{R}^{N}} f\left(x, u_{n}\right) u_{n}^{ \pm} d x=0
$$

Then, by (81) and $\lambda_{n} \rightarrow 1$, we have

$$
\begin{aligned}
\left\|u_{n}^{ \pm}\right\|^{2}= & \mp\left(1-\lambda_{n}\right) \int_{\mathbb{R}^{N}} V_{-}(x) u_{n} u_{n}^{ \pm} d x \\
& \mp \int_{\mathbb{R}^{N}} f\left(x, u_{n}\right) u_{n}^{ \pm} d x \\
\leq & C\left(1-\lambda_{n}\right)\left\|u_{n}\right\|_{L^{2}}^{2}+\left|\int_{\mathbb{R}^{N}} f\left(x, u_{n}\right) u_{n}^{ \pm} d x\right| \longrightarrow 0 .
\end{aligned}
$$

This contradicts $\inf _{n}\left\|u_{n}\right\|>0$ (see (88)). Therefore, case (a) cannot occur. As case (b) therefore occurs, $w_{n}=u_{n}\left(\cdot+y_{n}\right)$ satisfies $w_{n} \rightarrow u_{0} \neq 0$. From (14) and (43), we have that

$$
\Psi_{\lambda}(u)=-\Phi(u)+\frac{\lambda-1}{2} \int_{\mathbb{R}^{N}} V_{-} u^{2} d x, \quad \forall u \in X
$$

It follows that

$$
\begin{array}{r}
\left\langle\Psi_{\lambda}^{\prime}(u), \varphi\right\rangle=-\left\langle\Phi^{\prime}(u), \varphi\right\rangle+(\lambda-1) \int_{\mathbb{R}^{N}} V_{-} u \varphi d x, \\
\forall u, \varphi \in X .
\end{array}
$$

By $\Psi_{\lambda_{n}}^{\prime}\left(u_{n}\right)=0$ (see Lemma 12$)$, we have $\Psi_{\lambda_{n}}^{\prime}\left(w_{n}\right)=0$. From (106), we have that, for any $\varphi \in X$,

$$
\begin{aligned}
\left\langle\Psi_{\lambda_{n}}^{\prime}\left(w_{n}\right), \varphi\right\rangle= & -\left\langle\Phi^{\prime}\left(w_{n}\right), \varphi\right\rangle+\left(\lambda_{n}-1\right) \\
& \times \int_{\mathbb{R}^{N}} V_{-}(x) w_{n} \varphi d x .
\end{aligned}
$$

Together with $\Psi_{\lambda_{n}}^{\prime}\left(w_{n}\right)=0$ and $\lambda_{n} \rightarrow 1$, this yields

$$
\left\langle\Phi^{\prime}\left(w_{n}\right), \varphi\right\rangle \longrightarrow 0, \quad \forall \varphi \in X
$$

Finally, by $w_{n} \rightarrow u_{0} \neq 0$ and the weakly sequential continuity of $\Phi^{\prime}$, we have that $\Phi^{\prime}\left(u_{0}\right)=0$. Therefore, $u_{0}$ is a nontrivial solution of (1). This completes the proof.

\section{Conflict of Interests}

The authors declare that there is no conflict of interests regarding the publication of this paper.

\section{Acknowledgments}

The authors would like to thank the anonymous referees for their comments and suggestions on the paper. Shaowei Chen was supported by Science Foundation of Huaqiao University and Promotion Program for Young and Middle-Aged Teacher in Science and Technology Research of Huaqiao University (ZQN-PY119).

\section{References}

[1] N. Ackermann, "A nonlinear superposition principle and multibump solutions of periodic Schrödinger equations," Journal of Functional Analysis, vol. 234, no. 2, pp. 277-320, 2006.

[2] S. Alama and Y. Y. Li, "On "multibump" bound states for certain semilinear elliptic equations," Indiana University Mathematics Journal, vol. 41, no. 4, pp. 983-1026, 1992.

[3] V. Coti Zelati and P. H. Rabinowitz, "Homoclinic type solutions for a semilinear elliptic PDE on $\mathbb{R}^{n}$," Communications on Pure and Applied Mathematics, vol. 45, no. 10, pp. 1217-1269, 1992.

[4] S. Chen, "Multi-bump solutions for a strongly indefinite semilinear Schrödinger equation without symmetry or convexity assumptions," Nonlinear Analysis: Theory, Methods \& Applications, vol. 68, no. 10, pp. 3067-3102, 2008.

[5] Y. Ding and C. Lee, "Multiple solutions of Schrödinger equations with indefinite linear part and super or asymptotically linear terms," Journal of Differential Equations, vol. 222, no. 1, pp. 137-163, 2006.

[6] Y. Ding, Variational Methods for Strongly Indefinite Problems, vol. 7 of Interdisciplinary Mathematical Sciences, World Scientific Publishing Co. Pte. Ltd., Hackensack, NJ, USA, 2007.

[7] H.-P. Heinz, T. Küpper, and C. A. Stuart, "Existence and bifurcation of solutions for nonlinear perturbations of the periodic Schrödinger equation," Journal of Differential Equations, vol. 100, no. 2, pp. 341-354, 1992.

[8] L. Jeanjean, "On the existence of bounded Palais-Smale sequences and application to a Landesman-Lazer-type problem set on $\mathbb{R}^{N}$," Proceedings of the Royal Society of Edinburgh $A$ : Mathematics, vol. 129, no. 4, pp. 787-809, 1999.

[9] W. Kryszewski and A. Szulkin, "Generalized linking theorem with an application to a semilinear Schrödinger equation," Advances in Differential Equations, vol. 3, no. 3, pp. 441-472, 1998.

[10] W. Kryszewski and A. Szulkin, "Infinite-dimensional homology and multibump solutions," Journal of Fixed Point Theory and Applications, vol. 5, no. 1, pp. 1-35, 2009. 
[11] G. Li and A. Szulkin, "An asymptotically periodic Schrödinger equation with indefinite linear part," Communications in Contemporary Mathematics, vol. 4, no. 4, pp. 763-776, 2002.

[12] S. Liu, "On superlinear Schrödinger equations with periodic potential," Calculus of Variations and Partial Differential Equations, vol. 45, no. 1-2, pp. 1-9, 2012.

[13] A. A. Pankov and K. Pflüger, "On a semilinear Schrödinger equation with periodic potential," Nonlinear Analysis: Theory, Methods \& Applications, vol. 33, no. 6, pp. 593-609, 1998.

[14] A. Pankov, "Periodic nonlinear Schrödinger equation with application to photonic crystals," Milan Journal of Mathematics, vol. 73, pp. 259-287, 2005.

[15] W. Qin, J. Zhang, and F. Zhao, "Homoclinic orbits for a class of nonperiodic Hamiltonian systems," Abstract and Applied Analysis, vol. 2012, Article ID 769232, 20 pages, 2012.

[16] M. Schechter, "Superlinear Schrödinger operators," Journal of Functional Analysis, vol. 262, no. 6, pp. 2677-2694, 2012.

[17] M. Schechter and W. Zou, "Weak linking theorems and Schrödinger equations with critical Sobolev exponent," ESAIM Control, Optimisation and Calculus of Variations, vol. 9, pp. 601619, 2003.

[18] A. Szulkin and T. Weth, "Ground state solutions for some indefinite variational problems," Journal of Functional Analysis, vol. 257, no. 12, pp. 3802-3822, 2009.

[19] A. Szulkin and W. Zou, "Homoclinic orbits for asymptotically linear Hamiltonian systems," Journal of Functional Analysis, vol. 187, no. 1, pp. 25-41, 2001.

[20] C. Troestler and M. Willem, "Nontrivial solution of a semilinear Schrödinger equation," Communications in Partial Differential Equations, vol. 21, no. 9-10, pp. 1431-1449, 1996.

[21] M. Willem, Minimax Theorems, vol. 24 of Progress in Nonlinear Differential Equations and their Applications, Birkhäuser, Boston, Mass, USA, 1996.

[22] M. Willem and W. Zou, "On a Schrödinger equation with periodic potential and spectrum point zero," Indiana University Mathematics Journal, vol. 52, no. 1, pp. 109-132, 2003.

[23] M. Yang, "Ground state solutions for a periodic Schrödinger equation with superlinear nonlinearities," Nonlinear Analysis: Theory, Methods \& Applications, vol. 72, no. 5, pp. 2620-2627, 2010.

[24] M. Yang, W. Chen, and Y. Ding, "Solutions for periodic Schrödinger equation with spectrum zero and general superlinear nonlinearities," Journal of Mathematical Analysis and Applications, vol. 364, no. 2, pp. 404-413, 2010.

[25] M. Reed and B. Simon, Methods of Modern Mathematical Physics, Vol. I, Academic Press, New York, NY, USA, 1978.

[26] P. D. Hislop and I. M. Sigal, Introduction to Spectral Theory. With Applications to Schrödinger Operators, vol. 113 of Applied Mathematical Sciences, Springer, New York, NY, USA, 1996. 


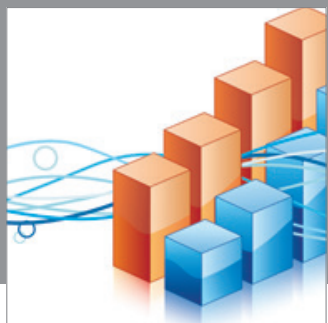

Advances in

Operations Research

mansans

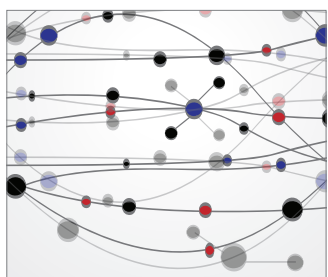

The Scientific World Journal
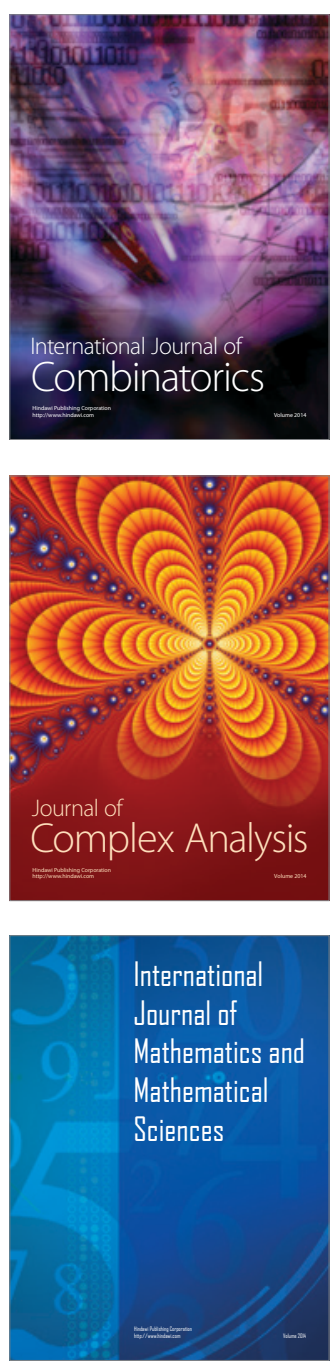


Submit your manuscripts at http://www.hindawi.com
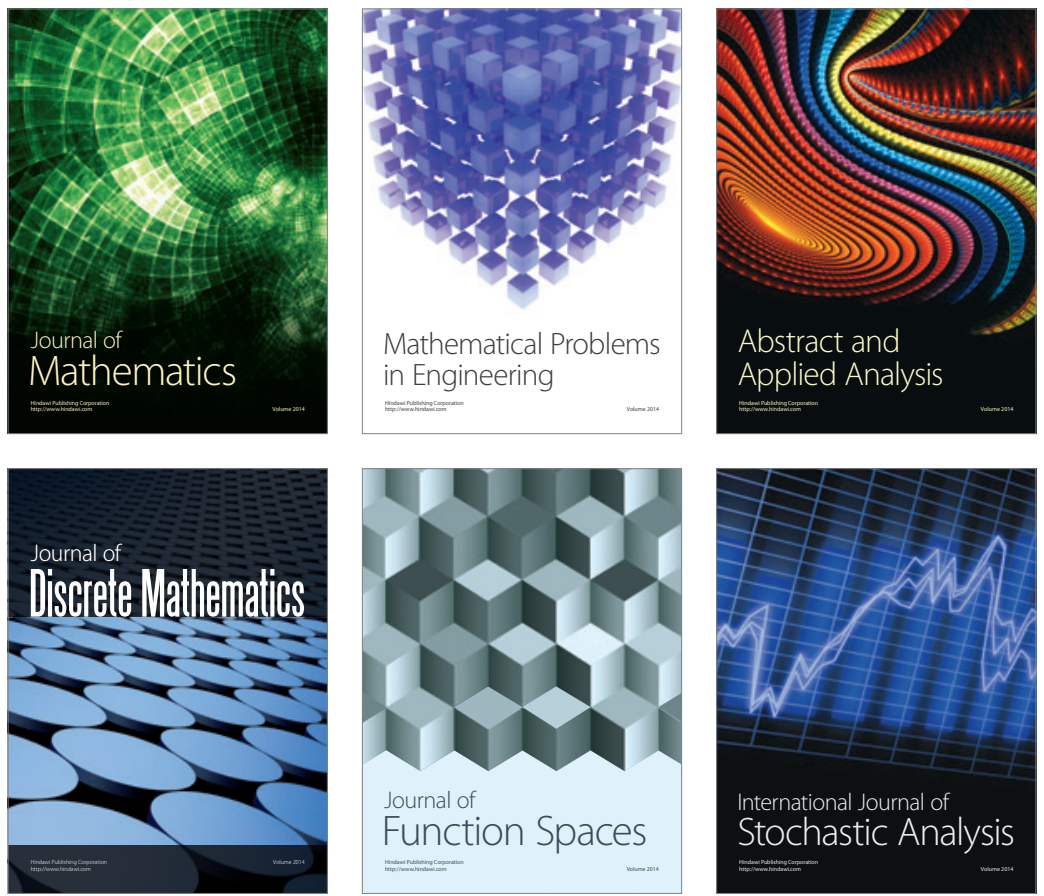

Journal of

Function Spaces

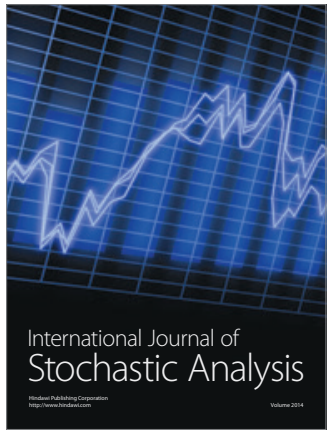

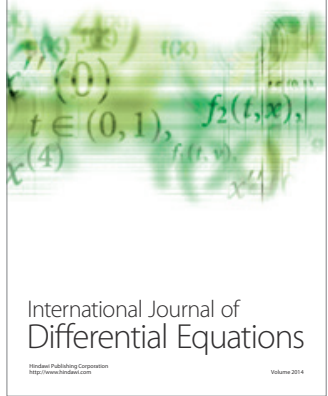
